\title{
Establishment of Anti-Human ATRX Monoclonal Antibody AMab-6
}

\author{
Satoshi Ogasawara, Yuki Fujii,, Mika K. Kaneko,, Hiroharu Oki, Hemragul Sabit, \\ Mitsutoshi Nakada, Hiroyoshi Suzuki, Koichi Ichimura, Takashi Komori, and Yukinari Kato ${ }^{1}$
}

Gliomas are the most frequently occurring brain tumors with a heterogeneous molecular background. The molecular subgrouping of gliomas more prognostically stratifies patients into distinct groups compared with conventional histological classification. The most important molecules for the subtype diagnosis of diffuse gliomas are mutations of isocitrate dehydrogenase (IDH), TERT promoter, and $\alpha$-thalassemia/mental-retardation-syndrome-X-linked (ATRX) and the codeletion of 1p/19q. Among them, IDH and ATRX mutations can be diagnosed using specific monoclonal antibodies (mAbs). We have developed many mAbs against IDH mutants, including HMab-1/HMab-2 against IDH1-R132H and multispecific mAbs MsMab-1/MsMab-2 against IDH1/2 mutations. In contrast, highly sensitive mAbs against ATRX remain to be established. In this study, we immunized mice with recombinant human ATRX and developed a novel mAb, AMab-6. The dissociation constant of AMab- 6 was determined to be $9.7 \times 10^{-10}$ $M$, indicating that the binding affinity of AMab-6 is very high. Furthermore, AMab-6 sensitively detects ATRX in Western blot and immunohistochemical analyses, indicating that AMab-6 could become the standard marker to determine the ATRX mutation status of gliomas in immunohistochemical analyses.

Keywords: ATRX, glioma, immunohistochemistry

\section{Introduction}

$\mathbf{T}$ HE MOLECULAR SUBGROUPING of gliomas using mutations of isocitrate dehydrogenase (IDH), $\alpha$-thalassemia/ mental-retardation-syndrome-X-linked (ATRX), and TERT promoter and the status of $1 \mathrm{p} / 19 \mathrm{q}$ more prognostically stratify patients into distinct groups compared with conventional histological classification. ${ }^{(1-4)}$ Treatment strategies can be planned with respect to molecular subtype along with the WHO grade; therefore, these molecular subtypes are clinically important. The inclusion of molecular parameters in the WHO definition of brain tumors was forwarded as the "ISN-Haarlem"' consensus. ${ }^{(5)}$ The 2016 WHO Classification of Tumors of the Central Nervous System (2016 CNS WHO) is both a conceptual and practical advance over the 2007 CNS WHO. The 2016 CNS WHO uses molecular parameters in addition to histology to define many tumor entities; presents major restructuring of the diffuse gliomas, medulloblastomas, and other embryonal tumors; and incorporates new entities, which are defined by both histology and molecular features. ${ }^{(6)}$
ATRX mutation in gliomas results in the loss of ATRX protein expression, which can be diagnosed by immunohistochemistry using anti-ATRX antibodies. ${ }^{(7)}$ Almost all studies of ATRX have used polyclonal antibodies ${ }^{(8)}$ because highly sensitive monoclonal antibodies (mAbs) against human ATRX remain to be established. Herein, we report a novel anti-ATRX $\mathrm{mAb}$, AMab-6, which is very useful in enzyme-linked immunosorbent assay (ELISA), Western blot, and immunohistochemical analyses.

\section{Materials and Methods}

\section{Cell lines and animals}

P3 U1 was obtained from the American Type Culture Collection (Manassas, VA) and was cultured in the RPMI 1640 medium (Nacalai Tesque, Inc., Kyoto, Japan), supplemented with $10 \%$ heat-inactivated fetal bovine serum (Thermo Fisher Scientific, Inc., Waltham, MA) at $37^{\circ} \mathrm{C}$ in a humidified atmosphere of $5 \% \mathrm{CO}_{2}$ and $95 \%$ air. Antibiotics, including $100 \mathrm{U} / \mathrm{mL}$ of penicillin, $100 \mu \mathrm{g} / \mathrm{mL}$ of streptomycin, and $25 \mu \mathrm{g} / \mathrm{mL}$ of amphotericin B (Nacalai Tesque, Inc.),

${ }^{1}$ Department of Regional Innovation, Tohoku University Graduate School of Medicine, Sendai, Japan.

${ }^{2}$ Department of Neurosurgery, Graduate School of Medical Science, Kanazawa University, Kanazawa, Japan.

${ }^{3}$ Department of Pathology and Laboratory Medicine, Sendai Medical Center, Sendai, Japan.

${ }^{4}$ Division of Brain Tumor Translational Research, National Cancer Center Research Institute, Tokyo, Japan.

${ }^{5}$ Department of Neuropathology, Tokyo Metropolitan Neurological Hospital, Tokyo, Japan.

(C) Satoshi Ogasawara et al., 2016; Published by Mary Ann Liebert, Inc. This Open Access article is distributed under the terms of the Creative Commons Attribution Noncommercial License (http://creativecommons.org/licenses/by-nc/4.0/) which permits any noncommercial use, distribution, and reproduction in any medium, provided the original author(s) and the source are credited. 
were added to the media. Female MRL/lpr mice ( 4 weeks old) were purchased from SLC Japan (Shizuoka, Japan). Animals were housed under pathogen-free conditions. The "Animal Care and Use Committee of Tohoku University" approved the animal experiments described herein.

\section{Hybridoma production}

Plasmid preparation and ATRX recombinant protein production are described in the Supplementary Data. MRL/lpr mice were immunized using an intraperitoneal (i.p.) injection of PA-ATRX-RAP-MAP $(100 \mu \mathrm{g})$ together with Imject Alum (Thermo Fisher Scientific, Inc.). After three additional immunizations, a booster injection was administered by i.p. 2 days before the spleen cells were harvested. The spleen cells were fused with P3 U1 cells using PEG1500 (Roche Diagnostics, Indianapolis, IN). The fused cells were grown in the RPMI medium with hypoxanthine, aminopterin, and thymidine selection medium supplement (Thermo Fisher Scientific, Inc.). Culture supernatants were screened using ELISA for the binding to recombinant MBP-ATRX-MAPPA or GST-ATRX-MAP-PA.

\section{Enzyme-linked immunosorbent assay}

Recombinant MBP-ATRX-MAP-PA or GST-ATRXMAP-PA was immobilized on Nunc MaxiSorp 96-well immunoplates (Thermo Fisher Scientific, Inc.) at a concentration of $5 \mu \mathrm{g} / \mathrm{mL}$ for 30 minutes. After blocking with $1 \%$ bovine serum albumin in $0.05 \%$ Tween 20/phosphatebuffered saline, the plates were incubated with culture supernatant followed by 1:3000-diluted peroxidase-conjugated anti-mouse IgG (Dako; Agilent Technologies, Inc., Glostrup, Denmark). The enzymatic reaction was performed with a 1Step Ultra TMB-ELISA (Thermo Fisher Scientific, Inc.). The optical density was measured at $655 \mathrm{~nm}$ using an iMark microplate reader (Bio-Rad Laboratories, Inc., Berkeley, CA).

\section{Determination of binding affinity using ELISA}

Purified recombinant protein was immobilized at $5 \mu \mathrm{g} / \mathrm{mL}$. The plates were incubated with serially diluted antibodies (26 pg/mL-10 $\mu \mathrm{g} / \mathrm{mL}$ ) followed by 1:1000-diluted peroxidaseconjugated anti-mouse IgG (Dako). The dissociation constant $\left(K_{\mathrm{D}}\right)$ was obtained by fitting the binding isotherms using the built-in one-site binding models in GraphPad Prism 6 (GraphPad Software, Inc., La Jolla, CA).

\section{Western blot analyses}

Recombinant protein $(0.1 \mu \mathrm{g})$ was boiled in SDS sample buffer (Nacalai Tesque, Inc.). The proteins were electrophoresed on 5\%-20\% polyacrylamide gels (Wako Pure Chemical Industries Ltd., Osaka, Japan) and were transferred onto a PVDF membrane (EMD Millipore Corp., Billerica, MA). After blocking with $4 \%$ skim milk (Nacalai Tesque, Inc.), the membrane was incubated with AMab-6 or antiMBP (clone: TMab-2; Wako Pure Chemical Industries Ltd.) and then with peroxidase-conjugated anti-mouse or anti-rat antibodies (1:1000 diluted; Dako) and developed with the Pierce Western Blotting Substrate Plus (Thermo Fisher Scientific, Inc.) or the ImmunoStar LD Chemiluminescence Reagent (Wako Pure Chemical Industries Ltd.) using the Sayaca-Imager (DRC Co. Ltd., Tokyo, Japan).

\section{Immunohistochemical analysis}

Surgical specimens of oligodendroglioma and diffuse astrocytoma were fixed in 4\% paraformaldehyde and embedded in paraffin for routine histopathological and immunohistochemical analysis. Immunohistochemical analysis was performed using avidin-biotin immunoperoxidase technique. In brief, 4- $\mu \mathrm{m}$-thick tissue sections were deparaffinized using xylene and rehydrated. After antigen retrieval using Target Retrieval Solution, pH 9.0 (Dako), the sections were autoclaved for 10 minutes and then maintained at room temperature for 40 minutes. Sections were quenched using $3 \%$ hydrogen peroxide $\left(\mathrm{H}_{2} \mathrm{O}_{2}\right)$ in methanol for 20 minutes and blocked with 5\% skim milk in TBST for 30 minutes. Sections were incubated with $3 \mu \mathrm{g} / \mathrm{mL}$ AMab- 6 overnight at $4^{\circ} \mathrm{C}$. Immunocomplexes were treated with an Envision+ Kit (Dako) for 1 hour. Color was developed using 3, 3-diaminobenzidine tetrahydrochloride (DAB Substrate Kit SK-4100, Vector, Japan) for 5 minutes. Sections were then counterstained with hematoxylin (Wako Pure Chemical Industries Ltd.). Images were acquired with a BZ-X700 microscope (Keyence, Osaka, Japan).

\section{Results and Discussion}

The two major methods for identifying these subtypes of gliomas are direct DNA sequencing and immunohistochemical analyses using antibodies. ${ }^{(9)}$ Immunohistochemistry is a
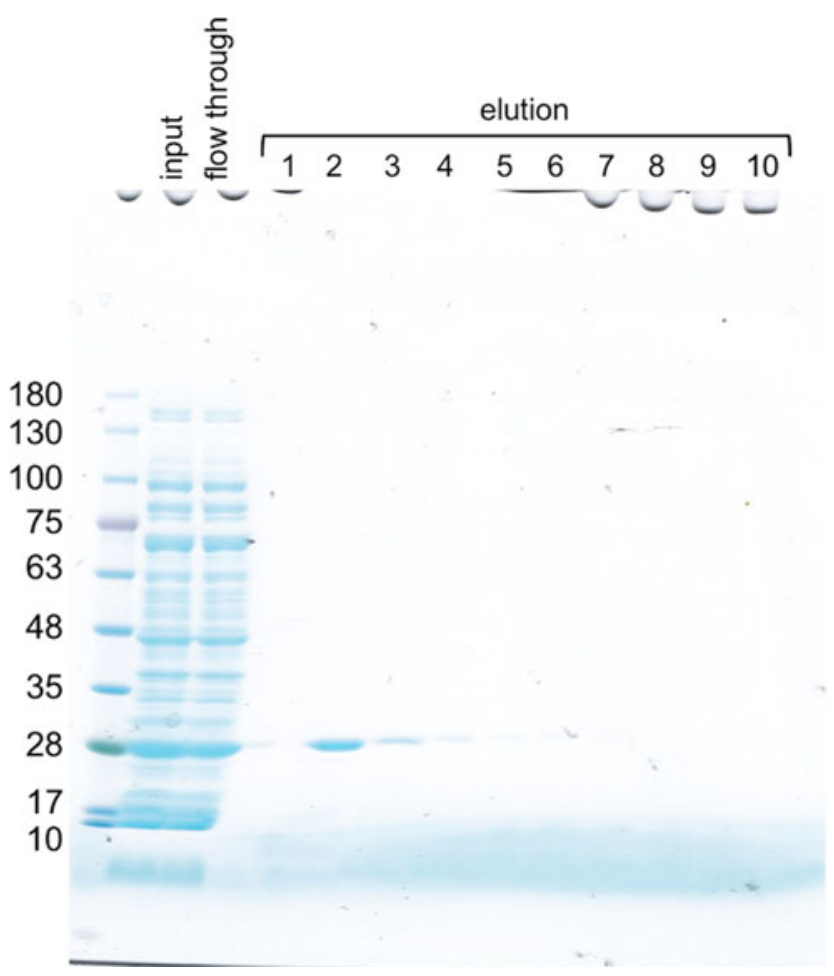

FIG. 1. Purification of the recombinant ATRX protein. Cleared lysate $(5 \mu \mathrm{L})$ from the transformed Escherichia coli before (input) and after (flow through) NZ-1-Sepharose capture, as well as 10 elution fractions (lanes 1-10) during column chromatography, was subjected to 5\%-20\% gradient SDS-PAGE under a reducing condition and stained with CBB. ATRX, $\alpha$-thalassemia/mental-retardation-syndrome$\mathrm{X}$-linked; SDS-PAGE, sodium dodecyl sulfate polyacrylamide gel electrophoresis. 
robust and widely available method, and immunohistochemical approaches with defined protocols and materials have become essential for assessing molecular genetic changes. ${ }^{(10)}$ Among many molecular parameters, the important molecules for subtype diagnosis of diffuse gliomas are mutations of IDH, TERT promoter, and ATRX and the codeletion of $1 \mathrm{p} / 19 \mathrm{q} .{ }^{(11)}$ In particular, mutations of IDH $^{(9)}$ and ATRX $^{(7,8)}$ can be accurately detected by immunohistochemical analysis. We developed many mAbs against IDH mutants, including HMab-1/HMab-2 against IDH1-R132H and multispecific mAbs MsMab-1/MsMab-2 against IDH1/2 mutations. ${ }^{(7,9,12-28)}$ However, highly sensitive mAbs against human ATRX remain to be established.

In this study, the recombinant PA-ATRX-RAP-MAP was captured using NZ-1-Sepharose and efficiently eluted with MES buffer ( $\mathrm{pH}$ 6.0), containing $3 \mathrm{M} \mathrm{MgCl}_{2}$ (Fig. 1). Mice were then immunized with PA-ATRX-RAP-MAP to develop novel anti-ATRX mAbs. We utilized the C-terminal region of ATRX as immunogen because commercially available polyclonal antibodies (Sigma-Aldrich) against ATRX were also produced against that region. ${ }^{(7)}$ Using ELISA, the culture supernatants of hybridomas were screened for binding to recombinant MBP-ATRX-MAP-PA or GST-ATRX-MAP-PA that was purified from Escherichia coli. As a result, AMab-6 (mouse $\mathrm{IgG}_{1}$, kappa) was established after limiting dilution.

As shown in Figure 2, AMab-6 reacted with MBP-ATRXMAP-PA in a dose-dependent manner. In contrast, AMab-6 did not react with MBP-IDH1. We further performed a kinetic analysis of the interaction of AMab- 6 with MBP-ATRX-MAP-PA (Fig. 2). The $K_{\mathrm{D}}$ of AMab-6 was determined to be $9.7 \times 10^{-10} \mathrm{M}$, indicating that the apparent binding affinity of AMab-6 is very high.

Western blot analysis demonstrated that AMab-6 detected MBP-ATRX-MAP-PA, but not MBP-IDH1, indicating that AMab-6 does not detect MBP tag in Western blot analysis (Fig. 3). Immunohistochemical analyses revealed that AMab-6 reacted with the nuclei of oligodendroglioma cells without ATRX mutations (Fig. 4A). ATRX mutations in gliomas result

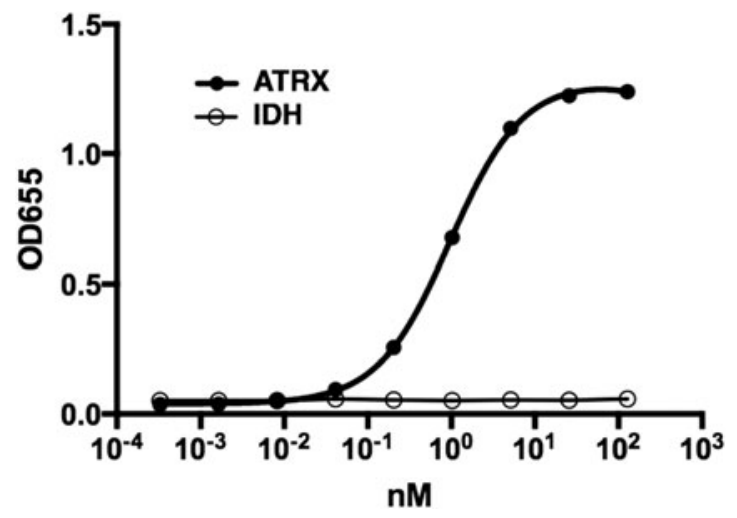

FIG. 2. Determination of binding affinity using enzymelinked immunosorbent assay. Purified recombinant protein (MBP-ATRX-MAP-PA or MBP-IDH1) was immobilized at $5 \mu \mathrm{g} / \mathrm{mL}$. The plates were incubated with serially diluted antibodies $(26 \mathrm{pg} / \mathrm{mL}-10 \mu \mathrm{g} / \mathrm{mL})$ followed by 1:1000-diluted peroxidase-conjugated anti-mouse IgG. The dissociation constant $\left(K_{\mathrm{D}}\right)$ was obtained by fitting the binding isotherms using the built-in one-site binding models in GraphPad Prism 6. IDH, isocitrate dehydrogenase.

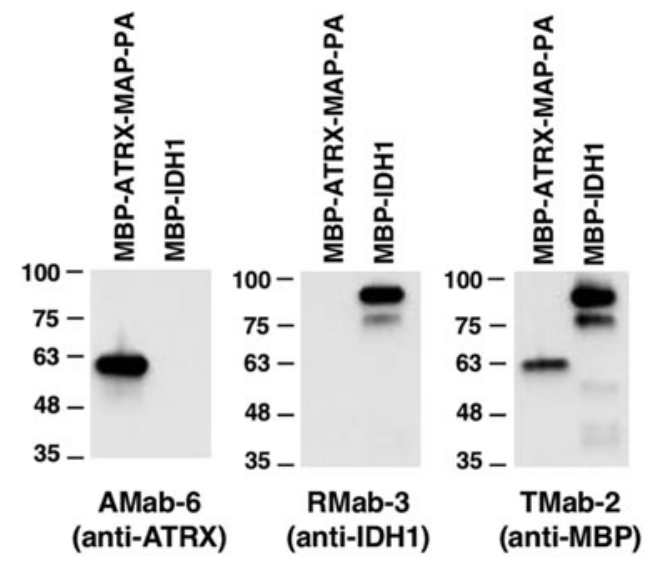

FIG. 3. Western blot analysis. Recombinant proteins $(0.1 \mu \mathrm{g})$ of MBP-ATRX-MAP-PA and MBP-IDH1 were electrophoresed and transferred onto a PVDF membrane. After blocking, the membrane was incubated with AMab-6, RMab-3 (anti-IDH1), or TMab-2 (anti-MBP).
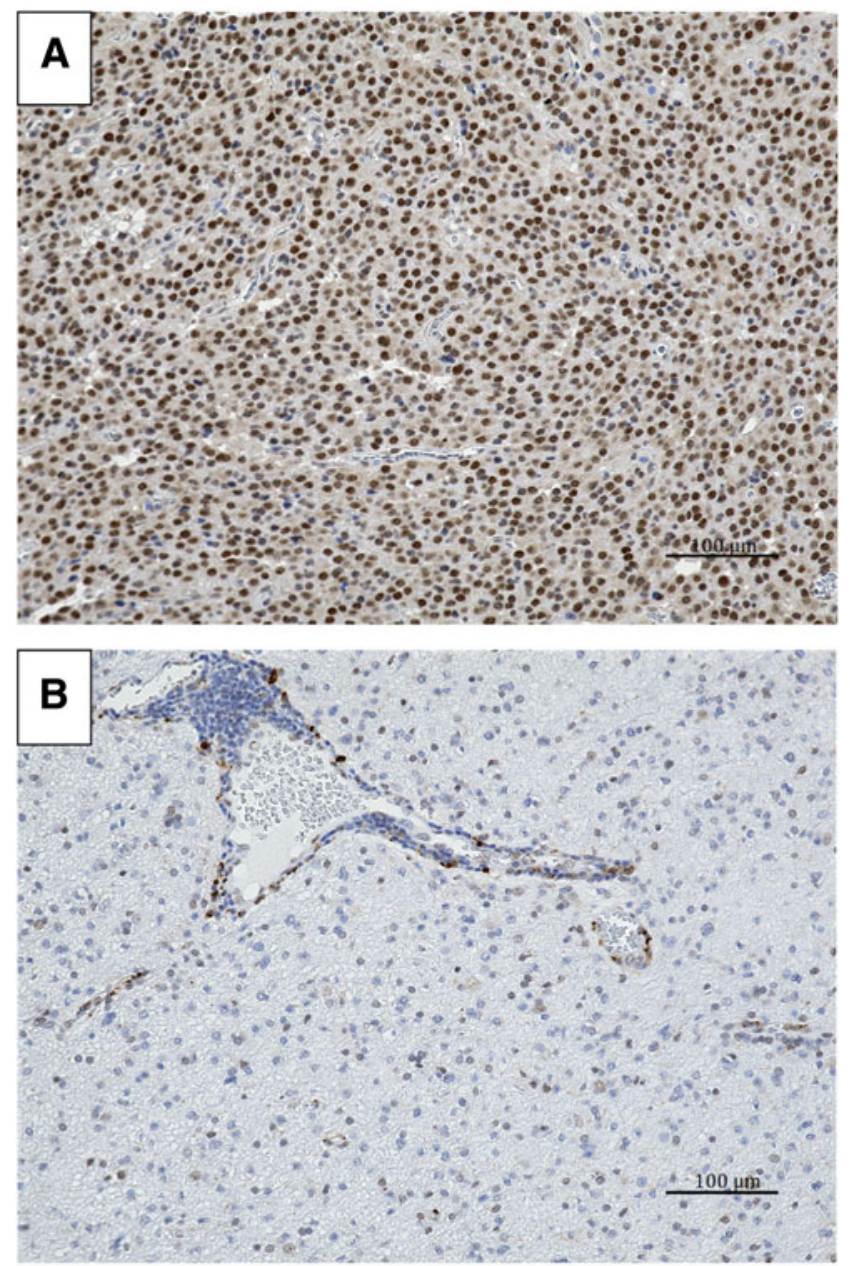

FIG. 4. Immunohistochemical analysis. (A) Oligodendroglioma, IDH-mutant (WHO grade II). Note the ATRX immunostaining in the nucleus of tumor cells without ATRX mutation. (B) Diffuse astrocytoma, IDH mutant (WHO grade II). Note the negative staining of ATRX in the nucleus of tumor cells with ATRX mutation, whereas positive staining was observed in the vascular endothelial cells without ATRX mutation. 
in the loss of ATRX protein expression, which can be diagnosed using immunohistochemical analysis. ${ }^{(7)}$ Likewise, AMab-6 did not recognize the nuclei of diffuse astrocytoma cells, which possess ATRX mutation, but reacted with those of vascular endothelial cells without ATRX mutation (Fig. 4B). These results indicate that AMab-6 is very sensitive against ATRX, which is expressed in gliomas without ATRX mutation.

In conclusion, a novel anti-ATRX mAb, AMab-6, sensitively reacted with human ATRX in ELISA, Western blot, and immunohistochemical analyses. Although many studies have used commercially available polyclonal anti-ATRX antibodies, ${ }^{(7)}$ we confirmed that the reactivity of AMab-6 is compatible with that of polyclonal antibodies (data not shown). Furthermore, we performed immunohistochemical analyses under many other conditions for antigen retrievals (citrate buffer, $\mathrm{pH} 6.0$ or 9.0) or incubation time of AMab-6 (30 minutes, 1 hour, or overnight) and obtained positive staining results as shown in Figure 4. Altogether, AMab-6 could serve as a standard marker to determine the ATRX mutation status of gliomas during immunohistochemical analyses.

\section{Acknowledgments}

The authors thank Takuro Nakamura, Noriko Saidoh, and Kanae Yoshida for their excellent technical assistance. This work was supported, in part, by the Practical Research for Innovative Cancer Control from Japan Agency for Medical Research and development, AMED (Y.K.); by JSPS KAKENHI Grant Number 25462242 (Y.K.) and 16K10748 (Y.K.), by the Regional Innovation Strategy Support Program from the Ministry of Education, Culture, Sports, Science and Technology (MEXT) of Japan (Y.K.), by the Platform for Drug Discovery, Informatics, and Structural Life Science (PDIS) from AMED (Y.K.), and by the Basic Science and Platform Technology Program for Innovative Biological Medicine from AMED (Y.K.). This work was performed, in part, under the Cooperative Research Program of Institute for Protein Research, Osaka University, CR-15-05 and CR-16-05.

\section{Author Disclosure Statement}

No competing financial interests exist.

\section{References}

1. Reuss DE, Sahm F, Schrimpf D, Wiestler B, Capper D, Koelsche C, Schweizer L, Korshunov A, Jones DT, Hovestadt V, Mittelbronn M, Schittenhelm J, Herold-Mende C, Unterberg A, Platten M, Weller M, Wick W, Pfister SM, and von Deimling A: ATRX and IDH1-R132H immunohistochemistry with subsequent copy number analysis and IDH sequencing as a basis for an "integrated" diagnostic approach for adult astrocytoma, oligodendroglioma and glioblastoma. Acta Neuropathol 2015;129:133-146.

2. Weller M, Weber RG, Willscher E, Riehmer V, Hentschel B, Kreuz M, Felsberg J, Beyer U, Loffler-Wirth H, Kaulich K, Steinbach JP, Hartmann C, Gramatzki D, Schramm J, Westphal M, Schackert G, Simon M, Martens T, Bostrom J, Hagel C, Sabel M, Krex D, Tonn JC, Wick W, Noell S, Schlegel U, Radlwimmer B, Pietsch T, Loeffler M, von Deimling A, Binder H, and Reifenberger G: Molecular classification of diffuse cerebral WHO grade II/III gliomas using genome- and transcriptome-wide profiling improves stratification of prog- nostically distinct patient groups. Acta Neuropathol 2015;129: 679-693.

3. Ceccarelli M, Barthel FP, Malta TM, Sabedot TS, Salama SR, Murray BA, Morozova O, Newton Y, Radenbaugh A, Pagnotta SM, Anjum S, Wang J, Manyam G, Zoppoli P, Ling S, Rao AA, Grifford M, Cherniack AD, Zhang H, Poisson L, Carlotti CG, Jr., Tirapelli DP, Rao A, Mikkelsen T, Lau CC, Yung WK, Rabadan R, Huse J, Brat DJ, Lehman NL, Barnholtz-Sloan JS, Zheng S, Hess K, Rao G, Meyerson M, Beroukhim R, Cooper L, Akbani R, Wrensch M, Haussler D, Aldape KD, Laird PW, Gutmann DH, Network TR, Noushmehr H, Iavarone A, and Verhaak RG: Molecular profiling reveals biologically discrete subsets and pathways of progression in diffuse glioma. Cell 2016;164:550-563.

4. Arita H, Narita Y, Yoshida A, Hashimoto N, Yoshimine T, and Ichimura K: IDH1/2 mutation detection in gliomas. Brain Tumor Pathol 2015;32:79-89.

5. Louis DN, Perry A, Burger P, Ellison DW, Reifenberger G, von Deimling A, Aldape K, Brat D, Collins VP, Eberhart C, Figarella-Branger D, Fuller GN, Giangaspero F, Giannini C, Hawkins C, Kleihues P, Korshunov A, Kros JM, Beatriz Lopes M, Ng HK, Ohgaki H, Paulus W, Pietsch T, Rosenblum M, Rushing E, Soylemezoglu F, Wiestler O, Wesseling $\mathrm{P}$, and International Society Of Neuropathology-Haarlem: International Society Of Neuropathology-Haarlem consensus guidelines for nervous system tumor classification and grading. Brain Pathol 2014;24:429-435.

6. Louis DN, Perry A, Reifenberger G, von Deimling A, Figarella-Branger D, Cavenee WK, Ohgaki H, Wiestler OD, Kleihues P, and Ellison DW: The 2016 World Health Organization Classification of Tumors of the Central Nervous System: A summary. Acta Neuropathol 2016;131:803-820.

7. Takano S, Ishikawa E, Sakamoto N, Matsuda M, Akutsu H, Noguchi M, Kato Y, Yamamoto T, and Matsumura A: Immunohistochemistry on IDH 1/2, ATRX, p53 and Ki-67 substitute molecular genetic testing and predict patient prognosis in grade III adult diffuse gliomas. Brain Tumor Pathol 2016;33:107-116.

8. Ebrahimi A, Skardelly M, Bonzheim I, Ott I, Muhleisen H, Eckert F, Tabatabai G, and Schittenhelm J: ATRX immunostaining predicts IDH and H3F3A status in gliomas. Acta Neuropathol Commun 2016;4:60.

9. Takano S, Tian W, Matsuda M, Yamamoto T, Ishikawa E, Kaneko MK, Yamazaki K, Kato Y, and Matsumura A: Detection of IDH1 mutation in human gliomas: Comparison of immunohistochemistry and sequencing. Brain Tumor Pathol 2011;28:115-123.

10. Tanboon J, Williams EA, and Louis DN: The diagnostic use of immunohistochemical surrogates for signature molecular genetic alterations in gliomas. J Neuropathol Exp Neurol 2016;75:4-18.

11. Suzuki H, Aoki K, Chiba K, Sato Y, Shiozawa Y, Shiraishi Y, Shimamura T, Niida A, Motomura K, Ohka F, Yamamoto T, Tanahashi K, Ranjit M, Wakabayashi T, Yoshizato T, Kataoka K, Yoshida K, Nagata Y, Sato-Otsubo A, Tanaka H, Sanada M, Kondo Y, Nakamura H, Mizoguchi M, Abe T, Muragaki Y, Watanabe R, Ito I, Miyano S, Natsume A, and Ogawa S: Mutational landscape and clonal architecture in grade II and III gliomas. Nat Genet 2015;47:458-468.

12. Kato Y: Specific monoclonal antibodies against IDH1/2 mutations as diagnostic tools for gliomas. Brain Tumor Pathol 2015;32:3-11.

13. Kato Y, Jin G, Kuan CT, McLendon RE, Yan H, and Bigner DD: A monoclonal antibody IMab-1 specifically recognizes 
IDH1R132H, the most common glioma-derived mutation. Biochem Biophys Res Commun 2009;390:547-551.

14. Kato Y, and Kaneko MK: Generation of a novel monoclonal antibody WMab-1 specific for IDH2-R172 W mutation. Biochem Biophys Res Commun 2013;433:374-378.

15. Kato Y, Natsume A, and Kaneko MK: A novel monoclonal antibody GMab-m1 specifically recognizes IDH1-R132G mutation. Biochem Biophys Res Commun 2013;432:564-567.

16. Kaneko MK, Morita S, Tsujimoto Y, Yanagiya R, Nasu K, Sasaki H, Hozumi Y, Goto K, Natsume A, Watanabe M, Kumabe T, Takano S, and Kato Y: Establishment of novel monoclonal antibodies KMab-1 and MMab-1 specific for IDH2 mutations. Biochem Biophys Res Commun 2013;432:40-45.

17. Kaneko MK, Tian W, Takano S, Suzuki H, Sawa Y, Hozumi Y, Goto K, Yamazaki K, Kitanaka C, and Kato Y: Establishment of a novel monoclonal antibody SMab-1 specific for IDH1-R132S mutation. Biochem Biophys Res Commun 2011;406:608-613.

18. Kaneko MK, Tsujimoto Y, Hozumi Y, Goto K, and Kato Y: Novel monoclonal antibodies GMab-r1 and LMab-1 specifically recognize IDH1-R132G and IDH1-R132 L mutations. Monoclon Antib Immunodiagn Immunother 2013;32: 224-228.

19. Liu X, Kato Y, Kaneko MK, Sugawara M, Ogasawara S, Tsujimoto Y, Naganuma Y, Yamakawa M, Tsuchiya T, and Takagi M: Isocitrate dehydrogenase 2 mutation is a frequent event in osteosarcoma detected by a multi-specific monoclonal antibody MsMab-1. Cancer Med 2013;2:803-814.

20. Takano S, Kato Y, Yamamoto T, Liu X, Ishikawa E, Kaneko MK, Ogasawara S, Matsuda M, Noguchi M, and Matsumura A: Diagnostic advantage of double immunohistochemistry using two mutation-specific anti-IDH antibodies (HMab-1 and MsMab-1) in gliomas. Brain Tumor Pathol 2015;32: 169-175.

21. Takano S, Kato Y, Yamamoto T, Kaneko MK, Ishikawa E, Tsujimoto Y, Matsuda M, Nakai K, Yanagiya R, Morita S, Tsuboi K, and Matsumura A: Immunohistochemical detection of IDH1 mutation, p53, and internexin as prognostic factors of glial tumors. J Neurooncol 2012;108:361-373.

22. Fujii Y, Ogasawara S, Oki H, Liu X, Kaneko MK, Takano S, and Kato Y: A high-sensitive HMab-2 specifically detects IDH1-R132H, the most common IDH mutation in gliomas. Biochem Biophys Res Commun 2015;466:733-739.
23. Ikota H, Nobusawa S, Arai H, Kato Y, Ishizawa K, Hirose $\mathrm{T}$, and Yokoo H: Evaluation of IDH1 status in diffusely infiltrating gliomas by immunohistochemistry using antimutant and wild type IDH1 antibodies. Brain Tumor Pathol 2015;32:237-244.

24. Liu X, Ogasawara S, Kaneko MK, Oki H, Hozumi Y, Goto K, Takagi M, and Kato Y: A novel monoclonal antibody SMab-2 recognizes endogenous IDH2-R172S of chondrosarcoma. Biochem Biophys Res Commun 2015;459:636-642.

25. Ohka F, Ito M, Ranjit M, Senga T, Motomura A, Motomura K, Saito K, Kato K, Kato Y, Wakabayashi T, Soga T, and Natsume A: Quantitative metabolome analysis profiles activation of glutaminolysis in glioma with IDH1 mutation. Tumour Biol 2014;35:5911-5920.

26. Sabit H, Nakada M, Furuta T, Watanabe T, Hayashi Y, Sato $\mathrm{H}$, Kato $\mathrm{Y}$, and Hamada J: Characterizing invading glioma cells based on IDH1-R132H and Ki-67 immunofluorescence. Brain Tumor Pathol 2014;31:242-246.

27. Ogasawara S, Kaneko MK, Tsujimoto Y, Liu X, and Kato Y: Multi-specific monoclonal antibody MsMab-2 recognizes IDH1-R132 L and IDH2-R172 M mutations. Monoclon Antib Immunodiagn Immunother 2013;32:377-381.

28. Kwon MJ, Kim ST, Kong DS, Lee D, Park S, Kang SY, Song JY, Nam DH, Kato Y, Choi YL, and Suh YL: Mutated IDH1 is a favorable prognostic factor for type 2 gliomatosis cerebri. Brain Pathol 2012;22:307-317.

Address correspondence to: Dr. Yukinari Kato Department of Regional Innovation Tohoku University Graduate School of Medicine 2-1 Seiryo-machi Aoba-ku Sendai Miyagi 980-8575 Japan

E-mail: yukinari-k@bea.hi-ho.ne.jp; yukinarikato@med.tohoku.ac.jp

Received: September 6, 2016 Accepted: September 15, 2016 